\title{
Carotid Doppler Evaluation of Transient Ischemic Attack And Stroke Patients And Its Correlation With CT Scan Head: A Prospective Study
}

\author{
Malik Rajesh ${ }^{1}$, Tiwari Richa ${ }^{2}$ \\ 1.Professor and Head of the Department, Department of Radiodiagnosis and Imaging, Gandhi Medical College \\ and Hamidia Hospital, Bhopal, India \\ 2.RMO third year, Department of Radiodiagnosis and Imaging, Gandhi Medical College and Hamidia \\ Hospital, Bhopal, India
}

\begin{abstract}
Sonographic evaluation of the carotid arteries, both gray scale and doppler is widely used for risk assessment for cerebrovascular accidents. Objective To establish the sensitivity of carotid doppler in patients with cerebrovascular accidents and the risk factors (on carotid USG) associated with severity of cerebrovascular accidents. Method- The present study Carotid Doppler evaluation of Transient Ischaemic Attack and Stroke patients and its correlation with CT scan head was carried out in 100 patients with cerebrovascular accidents during the period of September 2011 to September 2012. Patients with neurological symptoms were divided into two groups: stroke and transient ischaemic attack, based on CT head findings. All patients were then subjected to gray scale and doppler evaluation of carotid arteries. Results In stroke patients 42 out of 50 showed evidence of carotid stenosis (ipsilateral or bilateral) while 8 patients were normal. Thus the sensitivity of carotid doppler in stroke patients was $84 \%$. $70 \%$ of patients with $>50 \%$ stenosis had large infarct on CT scan head while only 25\% of patients with $<50 \%$ stenosis had large infarct ( $p$ value-0.003). Conclusion-Carotid doppler is an important non invasive diagnostic tool with high sensitivity
\end{abstract}

Keywords: Stroke, Transient ischaemic attack, Carotid gray scale, Carotid doppler, CT Head.

\section{Introduction}

Ischaemia due to flow limiting stenosis of the extracranial carotid arteries is common predisposing cause of stroke ${ }^{(1)}$. It has been documented that $80 \%$ of strokes are thromboembolic in origin, often with carotid plaque as the source of embolus ${ }^{(2,3)}$.Therefore early detection of these atheromatous changes in carotid artery will help a great deal in reducing stroke related morbidity and mortality. Sonographic evaluation of the carotid arteries, both Gray scale and Doppler is widely used for risk assessment. On Gray scale, we evaluate increased IMT (intima media thickness) in Common Carotid Artery which is considered by many as the earliest change of the atheromatous process ${ }^{(4)}$ Besides this, it helps to detects plaques, characterise them into those associated with high risk or low risk for cerebrovascular accidents, for example-those associated with intraplaque haemorrhage and surface ulceration are considered precursors of embolism and echogenic or calcified plaques are usually found in older, asymptomatic people ${ }^{(5)}$. Also reduction in carotid diameter evaluated in gray scale using transverse scan planes helps us find percentage stenosis. Colour Doppler evaluation shows areas of absent flow, especially useful in hypoechoic plaques not detectable on gray scale and allows detection of abnormal flow patterns. Spectral Doppler helps us to evaluate the waveform in carotid vessels, find peak Systolic velocity, end diastolic velocity, and abnormal characteristics like spectral broadening due to turbulent flow. Power. Doppler helps us detect colour streaks and helps differentiate between near occlusion and occlusion. Based on these criterion, we grade carotid stenosis into mild, moderate, severe, near occlusion and complete occlusion, which has got implications related to management. Trials like NASCET (North American Symptomatic Carotid Endarterectomy Trial) ${ }^{(6)}$ and ECST (European Carotid Surgery Trialists collaborative group) showed a definite benefit of carotid endarterectomy for patients with luminal narrowing of $>70 \%$ rather than for those below it ${ }^{(7)}$. Thus Doppler evaluation of the carotid artery has been shown to be cost effective, non invasive means of early detection of increased risk of CVAs with sensitivity approaching that of angiography.

\section{Objectives:}

Objective of this study was to evaluate stroke and transient ischaemic attack patients with carotid ultrasound for Intima Media thickness, presence and characterization of plaque (type, surface and site), spectral waveform analysis and percentage stenosis, to estabilish correlation between percentage stenosis and infarct size on CT scan head, to establish correlation between type, site of plaque and risk of infarction and to establish the sensitivity of carotid Doppler in patients of stroke. 
III. Methodology:

A prospective study of 100 cases of stroke and transient ischaemic attack was conducted in the Department of Radiodiagnosis and Imaging at Gandhi Medical College and Hamidia Hospital, Bhopal from September 2011 to September 2012

3.1 INCLUSION CRITERIA: Patients with symptoms of stroke and transient ischaemic attack such as transient episodes of neurological dysfunction, sudden weakness or numbness, hemiparesis, focal neurological deficits, sudden loss of consciousness, altered sensorium, aphasia, slurring of speech, diminution or loss of vision.

3.2 SOURCE OF DATA: Patients who presented in the Department of Medicine with clinical symptomatology of Transient Ischaemic Attack and stroke and were referred to Department of Radiodiagnosis at Gandhi Medical College and Hamidia Hospital for the purpose of CT scan head.

\subsection{EXCLUSION CRITERION:}

1) Evidence of posterior circulation stroke.

2) Evidence of haemorrhagic stroke.

\subsection{METHOD OF COLLECTION OF DATA:}

The study was approved by the Ethical and Research Committee of Gandhi Medical College and Hamidia Hospital, Bhopal. After finding the suitability as per inclusion and exclusion criteria, patients were selected for the study and briefed about the nature of the study, the interventions used and written informed consent was obtained. Further, descriptive data of the participants like name, age, sex, detailed history, were obtained by interviewing the participants and clinical examination and necessary investigations: CT scan head and gray scale and doppler evaluation of carotid arteries were recorded on predesigned and pretested proforma.

3.5 INSTRUMENTATION: All examinations were performed using GE Logic 3 Expert scanner with $5 \mathrm{MHz}$ transducer.

3.6 TECHNIQUE: The evaluation of carotid arteries was done with patient in supine position with neck extended and head turned to opposite side. Thorough gray scale examination was done for intima media thickness - done in longitudinal section at far wall of Common Carotid Artery and a mean of two values was taken. Presence, location and characterization (type and surface) of plaques were assessed. Measurement of diameter stenosis was done in transverse plane perpendicular to long axis of vessel. Spectral Doppler analysis was done in Common carotid artery, internal carotid artery and external carotid artery and measurements of Peak systolic velocity, end diastolic velocity were obtained on a frozen image and a mean of two values was taken. After this, Colour Doppler exam was done to substantiate the previous findings, identify regions of turbulence, any undetected areas of plaque or plaque ulceration. Depending upon the need, Power Doppler may or may not be employed to differentiate near complete obstruction from complete obstruction.

3.7 Statistical Methods:Data was compared by using unpaired ' $\mathrm{t}$ ' test and a 'p' value of $<0.05$ was considered significant.

\section{Results:}

Out of 100 patients of transient ischaemic attack and stroke, $64 \%$ were males and $36 \%$ were females. $46 \%$ patients were aged 61 years or above. Most common presentation was hemiparesis $(76 \%)$ followed by aphasia and cranial nerve palsy seen in $18 \%$ of patients each. Monoparesis was seen in $10 \%$ of cases while quadriparesis in $2 \%$ of them. Hypertension, smoking and Diabetes Mellitus were the three most common risk factors seen in 34\%, $32 \%$ and $30 \%$ of cases. Obesity, ischaemic heart disease, and hypercholesterolemia were other risk factors seen. Middle carotid artery territory was most frequently involved (92\% of stroke patients) while Anterior carotid artery territory was involved in $8 \%$. According to site, Basal Ganglia/ Internal Capsule region was the most commonly affected (76\% cases). Parietal, frontal and temporal lobes were affected in 52\%, $44 \%$ and $16 \%$ of patients respectively. Intima media thickness $>4 \mathrm{~mm}$ was not seen in Transient Ischaemic Attack patients, while it was seen in $2 \%$ of stroke patients. The mean Intima media thickness in Transient ischaemic attack and stroke patients was $1.1 \mathrm{~mm}$ and $1.73 \mathrm{~mm}$ respectively (Table 1). Multiple plaques were seen in $16 \%$ of Transient Ischaemic Attack patients and in $44 \%$ of stroke patients (Table 2), p value0.004(highly significant). Internal Carotid Artery was the most common site of plaque in stroke patients (43.7\%), while it accounted for $23 \%$ of plaques in transient ischaemic attack patients. p value- 0.04 (statistically significant). Common carotid artery was the most common site of plaque in Transient Ischaemic Attack patients 
(50\%). TYPE I and TYPE II were the most common types of plaques in stroke patients, together accounting for $56 \%$ of plaques. Calcified plaques (TYPE V) which don't embolize were the most common type in Transient ischaemic attack patients, accounting for $54.8 \%$ of plaques, while they accounted for just $12.5 \%$ of plaques in stroke patients, p value-0.00009(highly significant) (Table -3 ).

Most of the plaques (82\%) in Transient ischaemic attack patients were smooth while in stroke patients, approximately $46 \%$ plaques were smooth surfaced. Irregular plaques were much more commonly seen in stroke patients $(43.7 \%$ in stroke versus $18 \%$ in Transient ischaemic attack, $\mathrm{p}$ value-0.01(significant). Ulcerated plaques were seen exclusively in stroke patients $(9.3 \%)$. Majority of the plaques in Transient ischaemic attack patients $(72.7 \%$ ) caused $<50 \%$ stenosis, while just $28 \%$ plaques in stroke patients caused so. p value-0.00001(highly significant). Majority of plaques in stroke patients (43.7\%) caused 50-69\% stenosis. None of the plaques in TIA patients caused $70 \%$ or more stenosis while $28 \%$ of them in stroke patients caused so, p value- 0.0003 (highly significant) (Table - 4). There were minor differences in percentage of $<50 \%, 50-69 \%$ and 70 or more stenosis detected by colour doppler as compared to gray scale. (Table - 5). Most common types of plaques causing $<50 \%$ stenosis and 50-69\% stenosis in Transient Ischaemic Attack patients were type V and type II respectively. (Table -6 ). In stroke patients, Type I was the most common type of plaque causing $<50 \%$ stenosis while TYPE II was the most common type causing 50 or more stenosis (Table - 7). 16\% of stroke patients had no stenosis on carotid doppler evaluation. $60 \%$ of patients had ipsilateral carotid stenosis, $24 \%$ had bilateral stenosis. None had contralateral stenosis alone. Sensitivity of presence of carotid stenosis in stroke patients was $84 \%$. While $75 \%$ of patients with $<50 \%$ stenosis had small infarct on CT head, just $30 \%$ of patients with $>50 \%$ stenosis had small infarct, $p$ value- 0.003 (significant). $70 \%$ of patients with $>50 \%$ stenosis had large infarct on CT scan head while only $25 \%$ of patients with $<50 \%$ stenosis had large infarct, p value-0.003(significant). (Table-8)

TABLE - 1

INTIMA -MEDIA THICKNESS

\begin{tabular}{|c|c|c|}
\hline IMT & Transient ischaemic attack (\%) & Stroke (\%) \\
\hline$<1 \mathrm{~mm}$ & 60 & 18 \\
\hline $1-2 \mathrm{~mm}$ & 28 & 56 \\
\hline $2-3 \mathrm{~mm}$ & 8 & 14 \\
\hline $3-4 \mathrm{~mm}$ & 4 & 10 \\
\hline$>4 \mathrm{~mm}$ & 0 & 2 \\
\hline Total & 100 & 100 \\
\hline
\end{tabular}

TABLE - 2

NUMBER AND MULTIPLICITY OF PLAQUES

\begin{tabular}{|c|c|c|c|c|}
\hline \multirow{2}{*}{$\begin{array}{c}\text { No. of Plaques in a } \\
\text { patient }\end{array}$} & \multicolumn{2}{|c|}{ Transient ischaemic attack } & \multicolumn{2}{c|}{ Stroke } \\
\cline { 2 - 5 } & No. of Patients & $\%$ of patients & $\begin{array}{c}\text { No. of } \\
\text { Patients }\end{array}$ & $\%$ of patients \\
\hline None & 14 & 28 & 8 & 16 \\
\hline 1 & 28 & 56 & 20 & 40 \\
\hline 2 & 8 & 16 & 22 & 44 \\
\hline Total & 50 & 100 & 50 & 100 \\
\hline
\end{tabular}

TABLE - 3

DISTRIBUTION OF CASES BASED ON GRAY SCALE B MODE IMAGING-MORPHOLOGICAL GRADING OF ATHEROSCLEROTIC PLAQUE

\begin{tabular}{|c|c|c|c|c|}
\hline \multirow{2}{*}{\begin{tabular}{|} 
Type of plaque \\
Transient ischaemic attack
\end{tabular}} & \multicolumn{3}{c|}{ Stroke } \\
\cline { 2 - 6 } & No. of site & In $\%$ & No. of site & In $\%$ \\
\hline Type I & 4 & 9 & 16 & 25 \\
\hline Type II & 6 & 13.6 & 12 & 18.7 \\
\hline Type III & 6 & 13.6 & 8 & 12.5 \\
\hline Type IV & 4 & 9 & 8 & 12.5 \\
\hline Type V & 24 & 54.8 & 64 & 100 \\
\hline
\end{tabular}


TABLE-4

INCIDENCE OF CAROTID ARTERY STENOSIS BY GRAY SCALE B - MODE IMAGING

\begin{tabular}{|c|c|c|c|c|}
\hline \multirow{2}{*}{ Area \% stenosis } & \multicolumn{2}{|c|}{ Transient Ischaemic Attack } & \multicolumn{2}{c|}{ Stroke } \\
\cline { 2 - 5 } & No. of Sites & $\%$ & No. of Sites & $\%$ \\
\hline$<50$ & 32 & 72.7 & 18 & 28.1 \\
\hline $50-69$ & 12 & 27.3 & 28 & 43.7 \\
\hline 70 or more & - & - & 14 & 21.8 \\
\hline Near occlusion & - & - & 2 & 3.2 \\
\hline Total occlusion & - & - & 2 & 3.2 \\
\hline Total & 44 & 100 & 64 & 100 \\
\hline
\end{tabular}

TABLE - 5

Incidence Of Carotid Artery Stenosis By Colour Doppler

\begin{tabular}{|c|c|c|c|c|}
\hline \multirow{2}{*}{ Area \% stenosis } & \multicolumn{2}{|c|}{ Transient Ischaemic Attack } & \multicolumn{2}{c|}{ Stroke } \\
\cline { 2 - 5 } & No. of Sites & $\%$ & No. of Sites & $\%$ \\
\hline$<50$ & 30 & 68.2 & 16 & 25 \\
\hline $50-69$ & 14 & 31.8 & 30 & 46.9 \\
\hline 70 or more & - & & 14 & 21.9 \\
\hline Near occlusion & - & & 3 & 4.7 \\
\hline Total occlusion & - & & 1 & 1.5 \\
\hline TOTAL & 44 & 100 & 64 & 100 \\
\hline
\end{tabular}

TABLE - 6

Type Of Plaque Vs Degree Of Stenosis In Transient Ischaemic Attack Patients

\begin{tabular}{|c|c|c|c|c|c|c|c|c|c|c|c|}
\hline \multirow{3}{*}{$\begin{array}{l}\text { Percentage } \\
\text { Stenosis }\end{array}$} & \multicolumn{10}{|c|}{ TYPE OF PLAQUE } & \multirow{3}{*}{ TOTAL } \\
\hline & \multicolumn{2}{|l|}{$\mathrm{I}$} & \multicolumn{2}{|l|}{ II } & \multicolumn{2}{|l|}{ III } & \multicolumn{2}{|l|}{ IV } & \multicolumn{2}{|l|}{$\mathrm{V}$} & \\
\hline & No. & $\%$ & No. & $\%$ & No. & $\%$ & No. & $\%$ & No. & $\%$ & \\
\hline$<50$ & 2 & 6.2 & 0 & 0 & 6 & 18.7 & 4 & 12.5 & 20 & 62.5 & 32 \\
\hline $50-69$ & 2 & 16.6 & 6 & 50 & 0 & 0 & 0 & 0 & 4 & 33.4 & 12 \\
\hline 70 or more & - & & - & & - & & - & & - & & \\
\hline $\begin{array}{l}\text { Near } \\
\text { occlusion }\end{array}$ & - & & - & & - & & - & & - & & \\
\hline $\begin{array}{l}\text { Total } \\
\text { occlusion }\end{array}$ & - & & - & & - & & - & & - & & \\
\hline
\end{tabular}

TABLE - 7

TYPE OF PLAQUE VS DEGREE OF STENOSIS IN STROKE PATIENTS

\begin{tabular}{|c|c|c|c|c|c|c|c|c|c|c|c|}
\hline \multirow{3}{*}{$\begin{array}{l}\text { Percentage } \\
\text { Stenosis }\end{array}$} & \multicolumn{10}{|c|}{ TYPE OF PLAQUE } & \multirow{3}{*}{ TOTAL } \\
\hline & \multicolumn{2}{|c|}{ I } & \multicolumn{2}{|l|}{ II } & \multicolumn{2}{|l|}{ III } & \multicolumn{2}{|l|}{ IV } & \multicolumn{2}{|l|}{$\mathbf{V}$} & \\
\hline & No. & $\%$ & No. & $\%$ & No. & $\%$ & No. & $\%$ & No. & $\%$ & \\
\hline$<50$ & 6 & 33.3 & 2 & 11.1 & 4 & 22.2 & 4 & 22.2 & 2 & 11.1 & 18 \\
\hline $50-69$ & 6 & 21.4 & 12 & 42.8 & 4 & 14.2 & 2 & 7.1 & 4 & 14.2 & 28 \\
\hline 70 or more & - & - & 6 & 42.8 & 4 & 28.5 & 2 & 14.25 & 2 & 14.25 & 14 \\
\hline $\begin{array}{l}\text { Near } \\
\text { occlusion }\end{array}$ & - & - & 2 & 100 & - & - & - & - & - & - & 2 \\
\hline $\begin{array}{l}\text { Total } \\
\text { occlusion }\end{array}$ & 2 & 100 & - & - & - & - & - & - & - & - & 2 \\
\hline
\end{tabular}

TABLE -8 CORRELATION OF PERCENTAGE STENOSIS WITH SIZE OF INFARCT

\begin{tabular}{|l|l|l|l|}
\hline & \multicolumn{2}{|l|}{ INFARCT SIZE } & \\
\hline & SMALL & LARGE & TOTAL \\
\hline NO OR <50\%STENOSIS & $18(75 \%)$ & $6(25 \%)$ & 24 \\
\hline$>50 \%$ STENOSIS & $8(30 \%)$ & $18(70 \%)$ & 26 \\
\hline
\end{tabular}




\section{Discussion:}

Intima media thickness is considered as a surrogate marker for atherosclerotic disease not only in the cerebrovascular system, but in the whole arterial system ${ }^{(8)}$. It is believed that thickening of the intima media thickness complex greater than $0.8 \mathrm{~mm}$ is abnormal and may represent the earliest changes of atherosclerotic disease ${ }^{(9)}$.The mean intima media thickness in TIA and stroke patients was $1.1 \mathrm{~mm}$ and $0.82 \mathrm{~mm}$ respectively. The results showed that mean intima media thickness in our study was slightly greater than that found by Ratnakar Sahoo et al (2009) ${ }^{(10)}$, where it was $0.78 \mathrm{~mm}$ (range $0.5-1.5 \mathrm{~mm}$ ) in patient group and $0.594 \mathrm{~mm}$ (range $0.4-0.9 \mathrm{~mm}$ ) in controls. In this study $73 \%$ and $84 \%$ of patients showed evidence of plaques in transient ischaemic attack and stroke group of patients respectively. In a study by Muhammed -al -Najim et al ${ }^{(11)}, 63 \%$ of stroke patients had plaques while in that by Ratnakar Sahoo et al (2009) ${ }^{(10)}$, only $30 \%$ of patients had plaques. This may be due to differences in the study population. However, this also reflects the increased prevalence of carotid stenosis in ischaemic stroke patients. It can be explained by ever increasing incidence of atherosclerosis due to lifestyle changes and better management and control of other causes of ischaemic stroke like Rheumatic Heart Disease, infective endocarditis and vasculitis. The multiplicity of plaques was approximately thrice in stroke patients as compared to transient ischaemic attack patients. This may be explained by the more advanced stage of the disease in stroke patients.

Most of the plaques (81\%) in transient ischaemic attack patients were smooth surfaced while in stroke patients, irregular surfaced and ulcerated plaques together accounted for $53 \%$ of plaques. Similar results were obtained by Ulf Scheminke and Tillian Motsch et al (2000) ${ }^{(12)}$ This is because embolic occlusion of intracranial arteries is the primary cause of stroke and denuded or ulcerated carotid plaque surfaces are common sources of cerebral emboli. Therefore ulcerated plaques are important risk factor for ischaemic stroke. Majority of the plaques $(72.7 \%)$ caused $<50 \%$ stenosis in transient ischaemic attack patients while the majority $(43.7 \%)$ caused 50-69\% stenosis in stroke patients. None of the plaques in transient ischaemic attack patients caused $70 \%$ or more stenosis while $28 \%$ of them in stroke patients caused so. These finding correlated well with the study of Muhammed -al -Najim et al ${ }^{(11)}$ where patients with $>50 \%$ stenosis were associated with increased risk of ischaemic stroke than patients with lesser degrees of stenosis. This is because intraplaque haemorrhage and ulceration are considered unstable and lead to abrupt increases in plaque size. So larger plaques (stenosis greater than 70\%) were mostly Types 1 and 2 and typically found in symptomatic patients. Asymptomatic individuals with lesser degrees of stenosis generally have plaque type 4 and 5 . One patient misdiagnosed as total occlusion by colour Doppler was correctly diagnosed as near occlusion (with streak of residual lumen: string sign) on power Doppler. This is because power Doppler is more sensitive for detecting extremely low-amplitude, lowvelocity flow as the colour signal in power Doppler ultrasound is generated from the integrated power Doppler spectrum. Power Doppler ultrasound uses a larger dynamic range with a better signal-to-noise $(\mathrm{S} / \mathrm{N})$ ratio than colour Doppler ultrasound. Power Doppler sonography, unlike colour Doppler ultrasound, is largely angle independent. These features combine to make power Doppler ultrasound exquisitely sensitive to detecting a residual string of flow in the region of a suspected carotid occlusion.

Elevated Internal Carotid Artery Peak Systolic velocity was seen in all (100\%) patients with $>50 \%$ stenosis while elevated Internal Carotid Artery End Diastolic Velocity and Peak Systolic velocity Internal Carotid Artery/Common Carotid Artery lagged a bit behind correlating well with the study done by Hunik et $\mathrm{al}^{(13)}$. The increase in velocity in stenotic zone velocity is directly proportional to the severity of luminal narrowing. 42 out of 50 stroke patients showed evidence of carotid stenosis (ipsilateral alone or bilateral) while 8 patients were normal. Hence sensitivity of carotid Doppler in stroke patients $-84 \%$. In this study $75 \%$ of patients with $<50 \%$ stenosis had small infarct on CT head, while only $25 \%$ of these patients had large infarct.70\% of patients with $>50 \%$ stenosis had large infarct on CT scan head, just $30 \%$ of these patients had small infarct. This correlated well with the study of Muhammed -al -Najim et al ${ }^{(11)}$ where large infarcts were much more common in patients with $>50 \%$ stenosis than in patients with $<50 \%$ stenosis.

\section{Conclusion:}

The present study has shown that Carotid Doppler is an important non invasive diagnostic tool. It can be used for screening in high risk asymptomatic patients, patients with history of cerebrovascular events and for determining treatment protocol. Thus it should be used as a first line investigation in these patients supplemented by Magnetic Resonance Angiography whenever required and angiography should be used only in equivocal cases. 


\section{References.}

[1]. N R Sims and H Muyderman, Mitochondria oxidative metabolism and cell death in stroke, Biochimica et Biophysica Act,1802 (1),2009 80-91.

[2]. G A Donnan,M Fisher,M Macleod and S M Davis, Stroke,Lancet ,371 (9624),2008,1612-23.

[3]. A Shuaib and V C Hachinski , Mechanisms and management of stroke in the elderly ,CMAJ,45 (5),1991, 433-43

[4]. J F Polak, Intima-media thickness: a tool for atherosclerosis imaging and event prediction, The American journal of cardiology, 14,2002,244-69

[5]. M Sitzer, W Müller, M Siebler and W Hort, Plaque ulceration and lumen thrombus are the main sources of cerebral microemboli in high-grade internal carotid artery stenosis, Am Heart Assoc , 8, 1995, 456-61

[6]. Moneta et al, Correlation of North American Symptomatic Carotid Endarterectomy Trial (NASCET) angiographic definition of $70 \%$ to $99 \%$ internal carotid artery stenosis with duplexscanning,Journal of Vascular Surgery, 17 (1), 1993,152-159.

[7]. P M Rothwell and C P Warlow, Prediction of benefit from carotid endarterectomy in individual patients: a risk-modelling study, European Carotid Surgery Trialists Collaborative Group, Lancet ,353(9170), 1999,2105-2110.

[8]. J F Polak ,M J Pencina ,K M Pencina ,C J O'Donnell and P A Wolf,Carotid-wall intima-media thickness and cardiovascular events, $N$ Engl J Med,365(3),2011,213-21.

[9]. R Salonen,J T Salonen, Determinants of carotid intima-media thickness: a population-based ultrasonography study in Eastern Finnish men,Journal of Internal Medicine,229(3),1991, 225-231.

[10]. R Sahoo,V M Krishna,D K S Subrahmaniyan ,T K Dutta and S Elangovan, Common carotid intima-media thickness in acute ischaemic stroke: A case control study,Neurology India ,57,2009,627-630.

[11]. M M Al-Najim et al, Carotid Doppler In Stroke Patient, Stroke,38,2007,1655.

[12]. D Schminke and M Motsch , Three Dimensional Ultrasound observati on of carotid artery plaque ulceration,Stroke,28,1997, 343 347.

[13]. P Hunik P and C Barlar, Detection and Quantification of carotid artery stenosis: Efficacy of various Doppler parameters, AJR 160,1993, 619-625. 